\title{
Interaction of colloids with a nematic-isotropic interface
}

\author{
D. Andrienko, ${ }^{1}$ M. Tasinkevych, ${ }^{2}$ P. Patrício, ${ }^{2,3}$ and M. M. Telo da Gama ${ }^{2}$ \\ ${ }^{1}$ Max Planck Institute for Polymer Research, Ackermannweg 10, 55128 Mainz, Germany \\ ${ }^{2}$ Departamento de Física da Faculdade de Ciências and Centro de Física Teórica e Computacional, \\ Universidade de Lisboa, Avenida Professor Gama Pinto 2, P-1649-003 Lisboa Codex, Portugal \\ ${ }^{3}$ Instituto Superior de Engenharia de Lisboa, Rua Conselheiro Emídio Navarro 1, P-1949-014 Lisboa, Portugal
}

(Dated: October 30, 2018)

\begin{abstract}
The Landau-de Gennes free energy is used to calculate the interaction between long cylindrical colloids and the nematic-isotropic (NI) interface. This interaction has two contributions: one is specific of liquid crystals and results from the deformation of the director field close to the particles or to the interface, while the other is generic and results from wetting and surface tension effects.

Deep in the nematic phase the director field of long cylindrical colloids, with strong homeotropic anchoring, exhibits two half-integer defect lines. As the colloid moves towards the interface, the director configuration changes through a series of discontinuous transitions, where one or two of the defects are annihilated. In addition, the NI interface bends towards the colloid in order to minimize the elastic free energy in the nematic. In the isotropic phase, the colloid is surrounded by a thin nematic layer that reduces the surface free energy under favorable wetting conditions.

The interaction has a well-defined minimum near the interface. In this region the director and interfacial structures are complex and cannot be described analytically. Using the numerical results for the Landau-de Gennes free energy in the harmonic region, we obtained simple scaling laws for the (linear) force on the colloid.
\end{abstract}

PACS numbers: $61.30 . \mathrm{Cz}, 61.30 . J f, 61.20 . J a, 07.05 . \mathrm{Tp}$

\section{INTRODUCTION}

Colloidal dispersions are suspensions of solid or liquid particles in a host fluid. The size of the particles can vary from a few $\mathrm{nm}$ up to several $\mu \mathrm{m}$. Colloidal dispersions are often found in long-lived metastable states, providing the basis for a range of industrial applications, e. g. paints, drugs, foods, coatings, etc [1].

Colloidal dispersions in nematic liquid crystals form a special class of colloids. The difference from ordinary colloids arises from the long-range orientational order of the liquid crystal molecules, described by the so-called nematic director. Topological defects of the director field 2], additional long-range forces between colloidal particles 2, 3, 4], and, as a result, supermolecular structures [5, 6] are phenomena specific of colloidal nematics.

Colloidal nematics are usually prepared in the isotropic phase. To prevent flocculation due to attractive van der Waals forces, colloidal particles are treated to induce electrostatic or steric repulsive interactions. After cooling to a temperature below the NI transition, the colloids often segregate forming non-uniform clusters. In general, topological defects stabilize the dispersion [3] although, in some cases, they may help flocculation by producing additional attractive interactions [7].

The spatial distribution of colloidal particles is very sensitive to the cooling rate and cooling conditions [8, 9]. Recent results [10] revealed that the drag on colloids by a NI interface plays an important role in the final spatial pattern. In the experiment, large nematic and isotropic domains were separated by moving NI interfaces. Depending on the cooling rate (velocity of the interface) different structures were observed: a) cellular structures, also reported earlier 8, 9, 11], with particle-free nematic domains separated by particle-rich regions; b) striped structures, where the particle-rich regions are arranged in a set of stripes; c) root-like structures. A major conclusion of the study was that the motion of the particles may be controlled by their interaction with the NI interface.

Here we reexamine this conclusion by analyzing in detail the interaction between colloidal particles and a NI interface. As a first step we investigate the (static) Landau-de Gennes order parameter distributions around colloidal particles in the vicinity of NI interfaces. By calculating the corresponding free energies we obtain explicit results for the forces.

For simplicity, we consider the interaction of a long cylindrical colloid with a planar NI interface. We assume strong homeotropic anchoring of the director at the particle's surface everywhere and wetting boundary conditions (see section IIC). In the nematic phase, these anchoring conditions give rise to a pair of $1 / 2$ strength defect lines, that merge with the isotropic phase as the particle approaches the NI interface, distorting the planar interfacial region on a scale of the order of the colloidal radius. As a result, the interface is strongly bent around the colloidal particle on both sides of the interface. The effective interaction is shown to be rather complex and to exhibit a well defined minimum close to the interface. Near the minimum, a scaling analysis of the numerical results for the free energy yields the scaling form of the force on the particle.

The paper is organized as follows. The geometry, the model, and the minimization technique are described in Sec. III The results for the director configurations and 


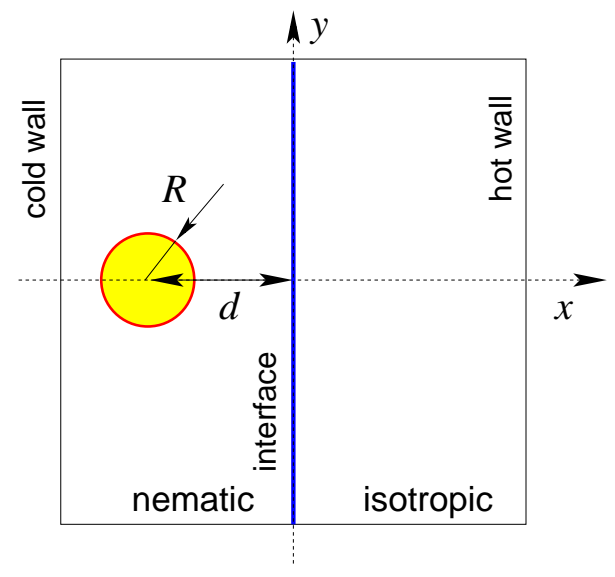

FIG. 1: $x y$ cross-section, of size $L \times L$, of the system studied.

free energy are presented in Sec. IIII A discussion of the major findings, together with concluding remarks, appear in Sec. IV]

\section{LANDAU-DE GENNES THEORY}

\section{A. Geometry}

In this study we consider a geometry similar to that used in the experiments [10]. Briefly, one end of the sample is placed in a hot and the other in a cold oven. The temperatures are chosen such that the NI interface is in the gap between the ovens and a constant temperature gradient is maintained throughout the sample.

A geometry mimicking the experimental setup is shown in Fig. 1. A uniform temperature gradient is imposed along the $x$ axis with the 'hot' wall at $x=L / 2$ and the 'cold' wall at $x=-L / 2$, where $L$ is the size of the system. The director at the 'cold' wall is fixed either parallel or perpendicular to the wall. The order parameter at the 'hot' wall is set to zero. Note, that the position of the interface, at $x=0$, is pinned by the temperature gradient.

A colloidal particle, which we take to be a long cylinder of radius $R$, with the symmetry axis parallel to the $z$ axis, is immersed in the nematic. Periodic boundary conditions are used in the $y$ direction.

\section{B. Tensor order parameter}

We use the Landau-de Gennes tensor order-parameter $Q_{\alpha \beta}$ formalism since it is free from divergent terms due to the defect cores and it takes into account the biaxiality that may occur in non-uniform nematics [12, 13, 14].

Owing to the traceless, symmetric character of the tensor order parameter it can be represented as [15]

$$
Q_{\alpha \beta}(\boldsymbol{r})=\frac{1}{2} Q\left(3 n_{\alpha} n_{\beta}-\delta_{\alpha \beta}\right)+\frac{1}{2} B\left(l_{\alpha} l_{\beta}-m_{\alpha} m_{\beta}\right),
$$

where the direction of maximal orientational order is given by the director $\boldsymbol{n}, Q$ is the scalar order parameter, and the unit vectors $\boldsymbol{l}, \boldsymbol{n}, \boldsymbol{m}$ form a local orthonormal triad.

Spatial non-uniformities that do not coincide with $\boldsymbol{n}$ break the cylindrical symmetry of the average angular environment, and thus one must allow for biaxiality, i.e. $B \neq 0$.

The translational symmetry of the interface along the $z$ axis and the assumed homeotropic boundary conditions on the colloidal surface, imply that the director is confined to the $x y$ plane. Then the vector $\boldsymbol{m}$ may be chosen along the $z$ axis and the vector $l$ is in the $x y$ plane

$$
\begin{aligned}
\boldsymbol{n} & =(\cos \theta, \sin \theta, 0), \\
\boldsymbol{l} & =(\sin \theta,-\cos \theta, 0), \\
\boldsymbol{m} & =(0,0,1) .
\end{aligned}
$$

In this case, the tensor order parameter has three independent components only

$$
\boldsymbol{Q}=\left(\begin{array}{ccc}
Q_{11} & Q_{12} & 0 \\
Q_{12} & Q_{22} & 0 \\
0 & 0 & -Q_{11}-Q_{22}
\end{array}\right)
$$

In the following we will use this representation of the tensor order parameter.

\section{Free energy}

The system is described by the Landau-de Gennes free energy [16]

$$
\mathcal{F}\{\boldsymbol{Q}\}=\int\left(f_{b}+f_{e}\right) d V+\int f_{s} d S
$$

where $f_{b}$ is the bulk free energy density, $f_{e}$ is the elastic free energy density and $f_{s}$ is the surface free energy. Within a mesoscopic approach the minimum of the Landau-de Gennes functional $\mathcal{F}$ gives the equilibrium value of the tensor order parameter.

Symmetry arguments yield for the local bulk free energy density [16, 17.

$$
f_{b}=a \operatorname{Tr} \boldsymbol{Q}^{2}-b \operatorname{Tr} \boldsymbol{Q}^{3}+c\left[\operatorname{Tr} \boldsymbol{Q}^{2}\right]^{2},
$$

where $a$ is assumed to depend linearly on the temperature, while the positive constants $b, c$ are taken temperature independent.

It is convenient to scale out the variables by defining

$$
\begin{gathered}
\tilde{Q}_{i j}=6 c / b Q_{i j}, \\
\tilde{f}_{b}=24^{2} c^{3} / b^{4} f_{b} .
\end{gathered}
$$

It will be understood that such scaling has been carried out, and we shall omit the overbars in the text below.

We also introduce a dimensionless temperature $\tau$ by defining

$$
a=\tau b^{2} / 24 c
$$


For a uniform uniaxial nematic $\left(Q_{11}=Q, Q_{22}=\right.$ $\left.Q_{33}=-1 / 2 Q\right)$ the free energy (5) takes the form

$$
f_{b}=\tau Q^{2}-2 Q^{3}+Q^{4}
$$

The nematic state is stable when $\tau<1$ with a degree of orientational order given by

$$
Q_{b}=\frac{3}{4}\left(1+\sqrt{1-\frac{8}{9} \tau}\right) .
$$

We modeled the temperature gradient in the $x$ direction by assuming that $\tau$ depends on the $x$ coordinate

$$
\tau=\tau_{c}\left(1+\alpha \frac{2 x}{L}\right)
$$

where $\tau_{c}=1$ is the NI transition temperature. Equation (10) implies that the NI transition occurs at $x=0$, i.e., the interface is in the middle of the cell and is parallel to the $y$ axis.

The elastic free energy density can be written as 17

$$
f_{e}=\frac{1}{2} L_{1} \frac{\partial Q_{i j}}{\partial x_{k}} \frac{\partial Q_{i j}}{\partial x_{k}}+\frac{1}{2} L_{2} \frac{\partial Q_{i j}}{\partial x_{j}} \frac{\partial Q_{i k}}{\partial x_{k}},
$$

where the constants $L_{1}$ and $L_{2}$ are related to FrankOseen elastic constants by $K_{11}=K_{33}=9 Q_{b}^{2}\left(L_{1}+\right.$ $\left.L_{2} / 2\right) / 2$ and $K_{22}=9 Q_{b}^{2} L_{1} / 2$ and $Q_{b}$ is the bulk nematic order parameter. The sign of $L_{2}$ defines the preferred orientation of the director at the NI interface. $L_{2}>0$ $\left(L_{2}<0\right)$ favors planar (perpendicular) anchoring [18].

We assumed strong homeotropic anchoring of the director at the colloidal surface. This is valid if the anchoring parameter $W R / K>>1$, where $W$ is the anchoring energy of the surface, and holds for large colloidal particles and/or anchoring strengths. We also assumed that the nematic at the particle surface is uniaxial with a scalar order parameter $Q_{\mathrm{s}}=1$, independent of $x$. Under these conditions, a planar interface is wetted by the nematic phase as the temperature is lowered through the NI transition.

\section{Minimization procedure}

The equilibrium distribution of the tensor order parameter $Q_{i j}$ is obtained by minimizing the free energy functional (4) numerically using finite elements with adaptive meshes. During the minimization the square integration region $L \times L$ was triangulated using the BL2D subroutine [19]. The functions $Q_{i j}$ are set at all vertices of the mesh and are linearly interpolated within each triangle. The free energy is then minimized using the conjugate gradients method [20] under the constraints imposed by the boundary conditions.

A new adapted mesh is generated iteratively from the previous minimization. The new local triangle sizes are calculated from the variations of the free energy, in order to guarantee a constant numerical weight for each

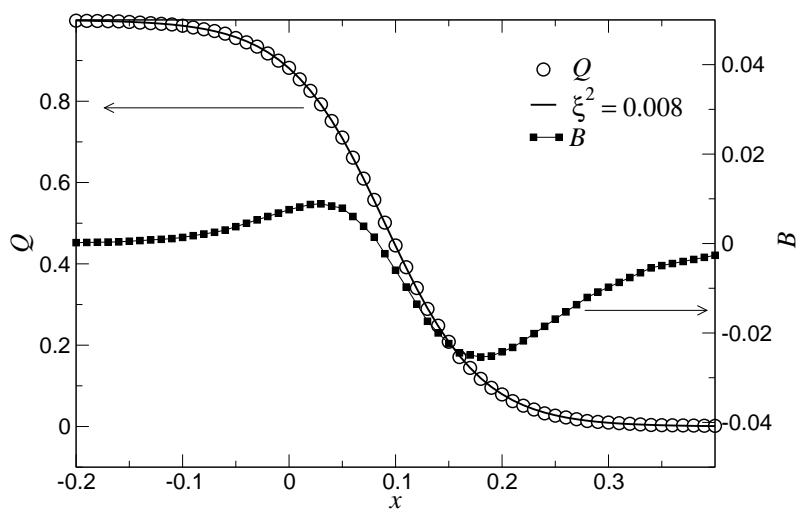

FIG. 2: Order parameter $Q$ and biaxiality $B$ of a free NI interface. Circles: order parameter, results of the numerical minimization; solid line: fit to Eq. (12). Squares: biaxiality, numerical results. Note the different scales and offset for the order parameter and biaxiality profiles.

minimization variable. The final meshes, with a minimal length of $\sim 10^{-2}$, had $\sim 10^{5}$ minimization variables. Lengths are in units of the diameter of the colloidal particle, $2 R=1$.

We used $\xi^{2}=L_{1} 8 c / b^{2}=0.001$ that corresponds to a nematic correlation length $\xi \approx 0.03$, which is larger than the smallest mesh length. The system size was either $L=12$ or $L=20$ and the temperature gradient was set at $\alpha=0.025$.

In order to obtain both stable and metastable configurations, we used different types of initial conditions including flat and curved NI interfaces.

\section{RESULTS}

\section{A. Free interface}

First, we analyze the tensor order parameter of a free NI interface, without colloidal particles and temperature gradients, for a system with $L_{2} / L_{1}=2$. For this ratio of the elastic constants the interface favors planar anchoring of the director (molecules parallel to the interface) 18]. The order parameter is fixed at the cell boundaries: $Q(x=-L / 2)=1, Q(x=L / 2)=0$ and the director is aligned along the $y$ axis, parallel to the interface.

The order parameter and biaxiality profiles are shown in Fig. 2 As expected, the order parameter changes from the bulk value of the nematic phase, $Q_{b}=1$, to the bulk value of the isotropic phase, $Q_{\text {iso }}=0$. The width of the interface is of the order of the nematic correlation length. The nematic and isotropic phases are uniaxial, with zero biaxiality. The interfacial region, however, is slightly biaxial.

Note, that the analytic solution of the corresponding variational problem does not exist, even in the one- 
dimensional case, when the tensor order parameter depends only on the $x$ coordinate. However, one may use the de Gennes Ansatz for the order parameter profile, which neglects biaxiality [18],

$$
Q=\frac{1}{2}\left(1-\tanh \frac{x}{\zeta}\right),
$$

where $\zeta$ is the nematic correlation length when the director is parallel to the interface

$$
\zeta^{2}=\xi^{2}\left(6+\frac{L_{2}}{L_{1}}\right) .
$$

A fit of the results of the numerical minimization to Eq. (12) yields $\zeta^{2} \approx 0.00807$ while Eq. (13) gives $\zeta^{2}=$ 0.008 , i.e., the agreement is very good. The small difference is attributed to the biaxiality of the interface [12], not taken into account by the Ansatz (12). Note that we decreased the minimal mesh length down to $10^{-3}$ in order to increase the accuracy of the results.

We also found that the surface tension is a function of the polar angle of the director with the interface normal. As a result, the interface has an easy axis with corresponding anchoring energy. We found planar interfacial anchoring (easy axis parallel to the interface) for $L_{2}>0$. $L_{2}<0$ results in homeotropic interfacial anchoring (easy axis normal to the interface) in agreement with previous results [12, 18].

We studied the interaction of a colloid with the NI interface under various anchoring conditions of the director at the interface: i) director parallel to the interface, $L_{2}>0$; ii) perpendicular to the interface, $L_{2}<0$; iii) director tilted with respect to the interface, due to a mismatch between interfacial anchoring and the alignment at the cell boundaries.

\section{B. Planar interfacial anchoring}

The anisotropy of the elastic constants is $L_{2} / L_{1}=2$, favoring director alignment parallel to the NI interface. The director at the 'cold' wall is fixed parallel the $y$ axis, which is also parallel to the NI interface.

Typical order parameter and director maps are shown in Fig. 3. The strong anchoring of the director at the colloidal surface yields two half-integer defect lines in the nematic, far from the interface, to ensure that the topological charge of the system is zero (see Fig. 3]). The director distortion vanishes very rapidly in the nematic phase, and the cores of the defects extend over a few nematic correlation lengths, in line with previous studies $[7,21,22$. At these distances there is no interaction between the colloid and the interface: the director at the interface is uniform, the interface is flat and it is pinned at $x=0$, where the nematic and isotropic phases coexist $(\tau=1)$.

On reducing the colloidal distance from the interface, the defect closest to the interface merges (discontinuously) with the isotropic phase (Fig. 3b,c). The interface
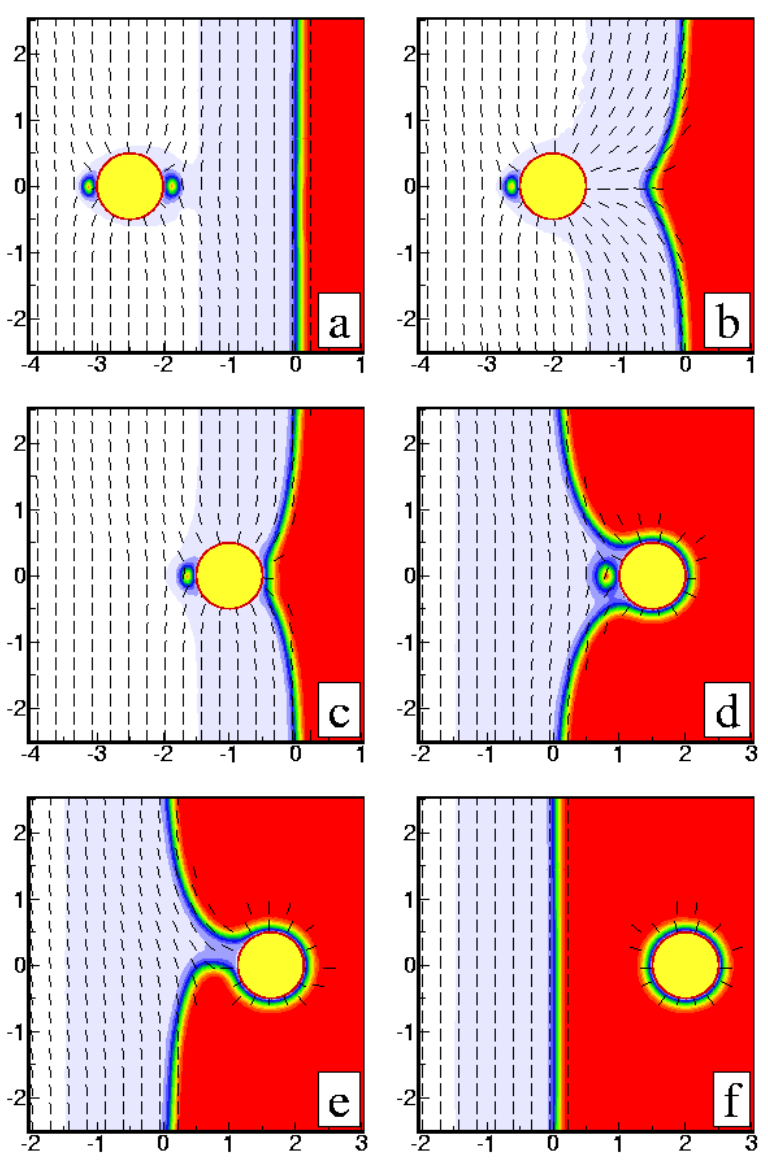

FIG. 3: Order parameter and director maps for colloids at a distance, $d$, from the NI interface: (a) $d=-2.5$; (b) $d=-2$; (c) $d=-1$; (d) $d=1.5$; (e) $d=1.625$; (f) $d=2$. Red (dark) corresponds to the isotropic phase with $Q=0$, and white to the nematic phase with $Q \approx 1$. System size $L=12$, anisotropy of the elastic constants $L_{2} / L_{1}=2$.

bulges towards the colloid to accommodate the isotropic phase, where the defect core disappeared. Note that the anchoring at the NI interface is planar and follows the interfacial curvature, except in the region where the defect used to be. Here the director tilts and the interfacial anchoring becomes homeotropic, see Fig. 3 b.

As the colloid moves further into the isotropic phase, it is wrapped by the NI interface that forms a nematic 'cavity' around the colloid (Fig. 31 d). The second defect is still present on the nematic side. At a certain point, this configuration becomes metastable, and eventually annihilation of the second defect occurs. This is accompanied by a symmetry breaking transition: the cavity is no longer symmetric under $y \rightarrow-y$ reflexion (Fig. 3 illustrates one of the two possible configurations). These configurations with broken symmetry are always metastable.

Finally, deep in the isotropic phase, the colloid is wrapped by a thin layer of nematic phase due to the (wetting) boundary conditions at the colloidal surface 


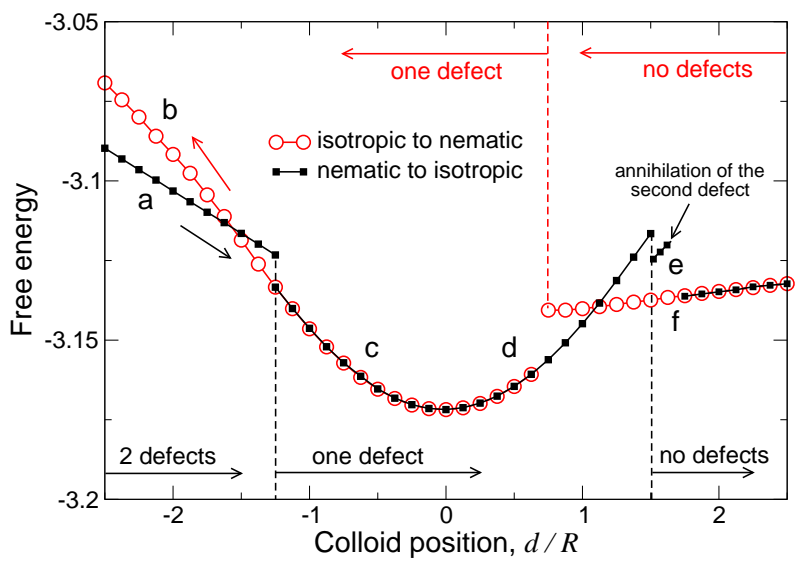

FIG. 4: Free energy $\mathcal{F}$ as a function of the distance of a colloidal particle from the NI interface. Both metastable and stable solutions are shown. Circles: particle moving from the isotropic to the nematic phase; squares: particle moving from the nematic to the isotropic phase. System size $L=$ 12 , anisotropy of the elastic constants $L_{2} / L_{1}=2$. Letters correspond to the director configurations shown in Fig. 3

\section{$\left(Q_{s}=1\right)$, Fig. B:}

To investigate the nature of the structural transitions between different director field configurations, we plot the free energy as a function of the particle position in Fig. 4

Deep in the isotropic phase, the configuration of Fig. 3: is the only stable one. There is a small force acting on the particle in the direction opposite to the temperature gradient. This force is due to an increase in the free energy of the nematic layer, as the particle moves into a region at higher temperature.

Moving from the isotropic into the nematic phase, this configuration becomes first metastable, and then unstable. At a certain point, the configuration with a nematic 'cavity' in the isotropic phase, Fig. 31, becomes stable.

Finally in the nematic phase, the configuration with one defect becomes metastable, and then unstable. The second defect appears, as shown in Fig. 3 a . At this point the interaction of the particle with interface almost vanishes. The small negative slope of the free energy, giving rise to a force along the temperature gradient, is due to the temperature dependence of the elastic energy, that increases as the particle moves towards the cold wall.

The jumps in the free energy reveal discontinuous (or first-order) instabilities between the physical director configurations. The equilibrium position, i.e., the position where the force on the colloid vanishes, is near the interface, at $x=0$.

\section{Homeotropic interfacial anchoring}

Liquid crystals with negative elastic constant anisotropy, $K_{22}>K_{11}, K_{33}$, are modeled by $L_{2} / L_{1}<0$
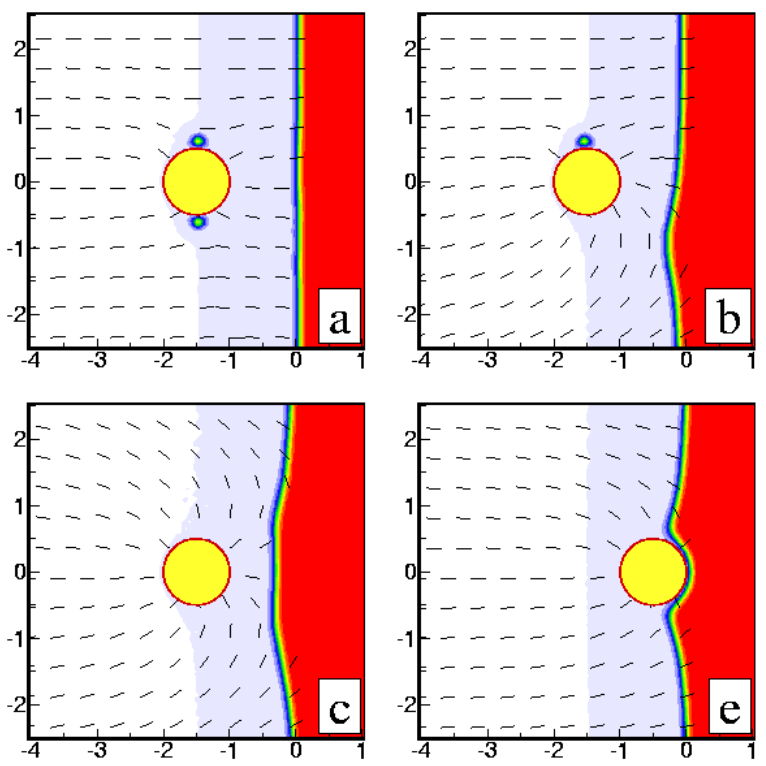

FIG. 5: Order parameter and director maps for colloids at a distance, $d$, from the NI interface: $(\mathrm{a}, \mathrm{b}, \mathrm{c}) d=-1.5$; (e) $d=-0.5$. System size $L=20$, anisotropy of the elastic constants $L_{2} / L_{1}=-0.5$.

[16]. In these systems the NI interface favors homeotropic anchoring, i.e., alignment of the molecules perpendicular to the interface [18].

To study this case, we fixed the director at the cold wall along the $x$ axis and used for the elastic constants $L_{2} / L_{1}=-0.5$. For this set of parameters the director is parallel to the $x$ axis, or perpendicular to the interface.

The order parameter and director maps are shown in Fig. [5] Since the director is perpendicular to the interface, the defect lines are in the plane parallel to the interface, Fig. [5]. In this geometry both defects merge with the isotropic phase very quickly, when the particle is still on the nematic side of the interface, Fig. 5 . Subsequently, the particle is surrounded by a thin layer of nematic phase until the fully wrapped state in the isotropic phase obtains, Fig. 3:

Deep in the nematic phase, we have also found a configuration with only one defect, Fig. 55. However, analysis of the free energy, Fig. [6] shows that this configuration is always metastable.

\section{Director tilted at the NI interface}

Another situation, often found in experiments, corresponds to tilted NI interfaces. However, the (simple) Landau-de Gennes theory fails to describe this interfacial anchoring and higher order gradients or more sophisticated density-functional theories are required in order to describe tilt at NI interfaces [23]. In finite cells, on the other hand, tilted interfaces may result from mismatch- 


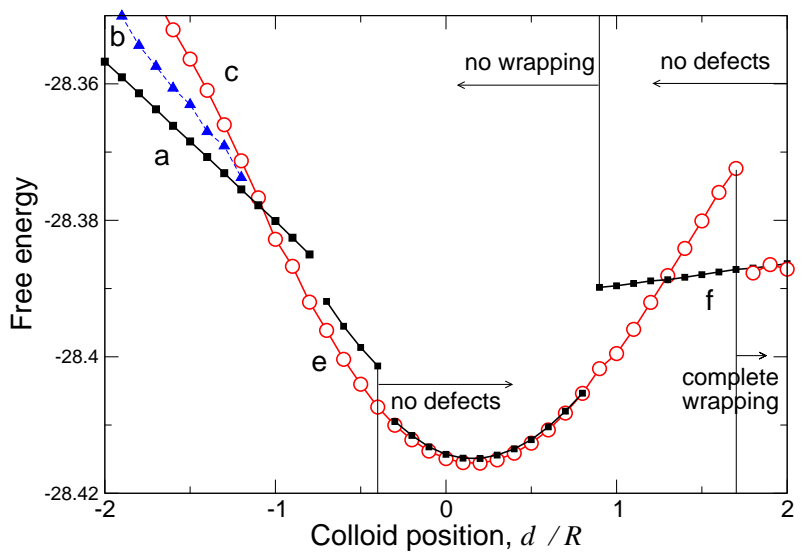

FIG. 6: Free energy $\mathcal{F}$ as a function of the distance of a colloidal particle from the NI interface. Squares: initial condition with a 'flat' interface; circles: initial condition with a 'curved' interface; triangles: 'curved' interface with the initial director tilted at $\pi / 4$ (metastable configuration). System size $L=20$, anisotropy of the elastic constants $L_{2} / L_{1}=-0.5$. Letters correspond to the director configurations shown in Fig. 5

ing boundary conditions at the cell boundaries and the NI interface proper. Indeed, in a planar cell, one may have the director aligned parallel to the bounding plates, say, along the $x$ axis and a NI interface in the $z y$ plane providing planar anchoring along the $z$ axis. This is the socalled hybrid, or $\pi$-cell 24, 25] where the director bends in the bulk to match the boundary conditions; as a result there is a bulk splay-bend deformation even in the absence of colloidal particles.

For this geometry we used $L_{2} / L_{1}=2$ and fixed the director at the 'cold' wall parallel to the $x$ axis or perpendicular to the NI interface. The system size is $L=20$.

The order parameter and director maps, shown in Fig. 7 are qualitatively similar to those described in section IIIB where the director is parallel to the cold wall and the interface. The tilt of the defects is due to the tilted nematic director, Fig. 7 7 . Otherwise, the same configurations are present: with two defects, deep in the nematic phase (Fig. 7 ); with one defect, close to the interface (Fig. 17b,d); without defects, also close to the interface (Fig. 76); without defects, deep in the isotropic phase (similar to the configuration shown in Fig. 3if).

However, a careful analysis of the free energy, which is shown in Fig. 8 reveals that the structural transitions are different. The configuration with one defect (Fig. [7d) becomes metastable in the isotropic side of the interface; the defect-free configuration (Fig. 7f), that was metastable previously, is now stable. A physical explanation is simple: because of the tilt, the remaining defect is closer to the interface and it is more easily annihilated. The bending of the interface is also weaker.
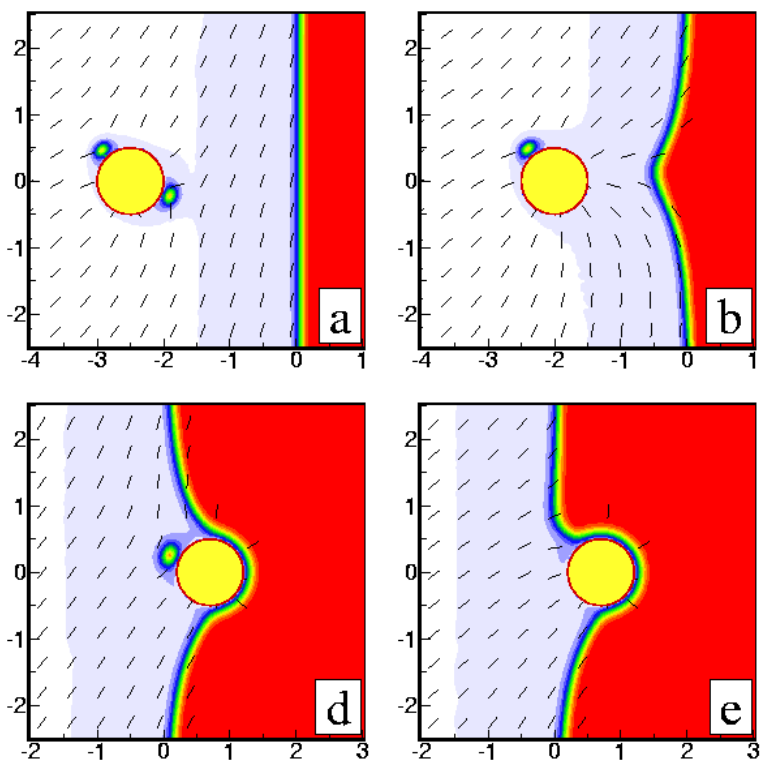

FIG. 7: Order parameter and director maps for colloids at a distance, $d$, from the NI interface: (a) $d=-2.5$; (b) $d=-2$; (d,e). Red (dark) corresponds to the isotropic phase with $Q=0$ and white to the nematic phase with $Q \approx 1$. System size $L=20$, anisotropy of the elastic constants $L_{2} / L_{1}=2$.

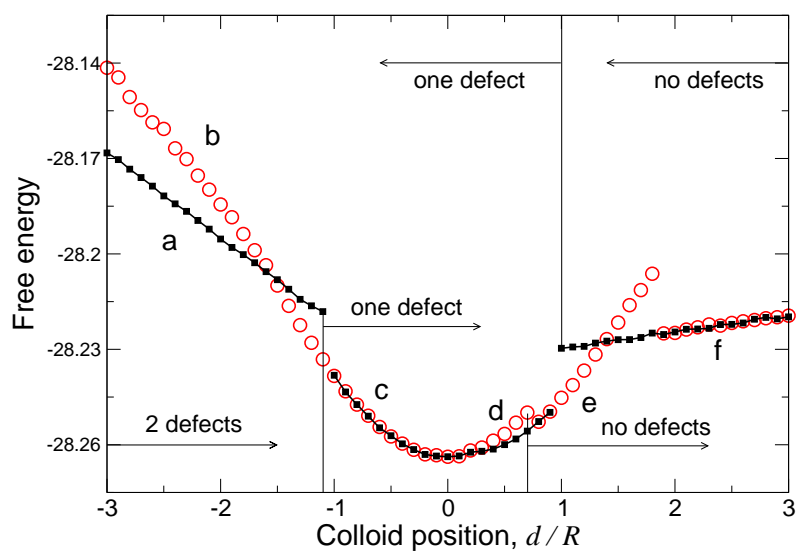

FIG. 8: Free energy $\mathcal{F}$ as a function of the distance of a colloidal particle from the NI interface. Squares: initial condition with a 'flat' interface; circles: initial condition with a 'curved' interface. System size $L=20$, anisotropy of the elastic constant $L_{2} / L_{1}=2$. The director is tilted at the NI interface. Letters correspond to the director configurations shown in Fig. 7

\section{DISCUSSION AND CONCLUSIONS}

We end with a brief discussion of the implications of our work on the drag of colloidal particles by the NI interface.

The free energy, Figs. 4 [6] and 8 reveals that close 


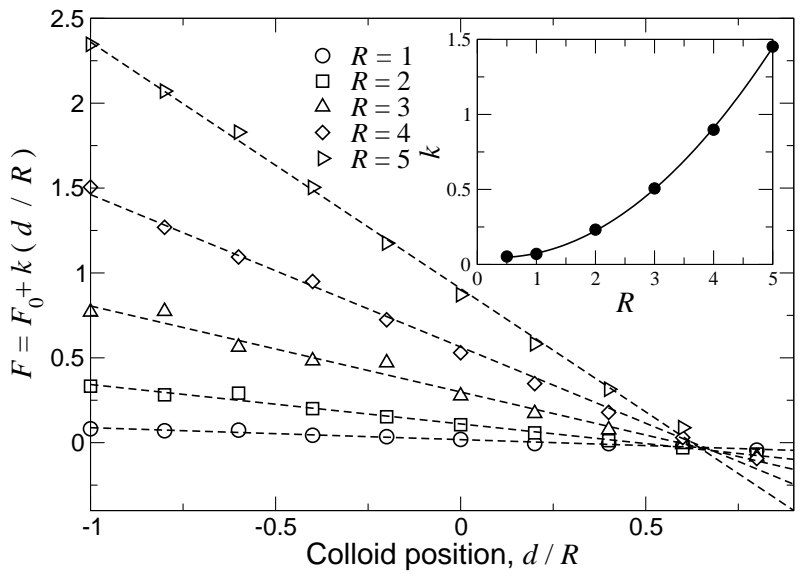

FIG. 9: Force on the particle as a function of the particle position. Inset: strength of the force as a function of the colloidal size and fit to $k \propto R^{2}$.

to the minimum $\mathcal{F}$ is a quadratic function of the position of the colloid and thus, in this region, the force is proportional to the separation, $d$.

We have calculated the force $F=-\partial \mathcal{F} / \partial d$, as a function of $d$, for several colloidal sizes, in the geometry with planar interfacial anchoring, as in the section $11 \mathrm{~B}$ The resulting curves, together with the fit to the following dependence

$$
F=F_{0}+k \frac{d}{R}
$$

are shown in Fig. 9 The inset of Fig. 9 shows the dependence of the force strength $k$ on the particle size. We find that, to a good approximation, $k \propto R^{2}$.

It is interesting to compare this result to the scaling arguments used in Ref. [10]. If we neglect phenomena related to the creation and annihilation of defects, and treat the interface as sharp, the only parameters in the problem are the radius and length of the colloid, $R$ and $L$ respectively, the elastic constant $K$, and the surface tension $\sigma$. The appropriate combinations of these parameters with the dimensions of force are $K L / R$ and $\sigma L R$, where we took into account that for a particle of length $L>>R$ the force is proportional to $L$. Therefore, the elastic and the surface tension contributions to the total force can be written as $F_{\mathrm{e}}=K L / R f_{\mathrm{e}}(d / R), F_{\mathrm{s}}=L \sigma f_{\mathrm{s}}(d / R)$, where $f_{\mathrm{e}}$ and $f_{\mathrm{s}}$ are dimensionless functions of the penetration depth, $d / R$. It is clear that neither of these forces gives the observed $\propto R^{2}$ dependence on the particle size: the elastic force is inversely proportional to $R$, while the contribution due to the surface tension does not depend on the particle size at all. One reason for this discrepancy (apart from the presence of defects) is the complex structure of the interface, that bends forming a cavity to wrap the colloid.

Another interesting conclusion is the asymmetry of the free energy profile as a function of the colloid position: when the particle moves from the nematic to the isotropic phase, the metastable phase with a nematic 'cavity' (Fig. 3i) becomes unstable much faster than the corresponding metastable state with one or no defects (Figs. 3b), when the particle moves from the isotropic to the nematic phase. Therefore, the interface drags the particle more efficiently when the colloid moves from the nematic to the isotropic side, owing to the presence of a higher energy barrier. This effect has been observed experimentally 10 .

We also note that wrapping a colloidal particle by the $\mathrm{NI}$ interface resembles the process of wrapping a colloidal particle by a membrane [26, 27]. A detailed comparison of these processes requires, however, further analysis.

To summarize, we studied the interaction of a cylindrical colloidal particle with a NI interface. We found that, when the particle is close to the interface, the force on the particle is proportional to the penetration depth $d / R$, and the amplitude of the force scales as $R^{2}$. At larger penetrations, discontinuous configurational transitions occur, related to the creation/annihilation of topological defects.

\section{Acknowledgments}

It is pleasure to thank M. Deserno, Yu. Reznikov, and A. Glushchenko for stimulating discussions. DA acknowledges the support of the Alexander von Humboldt foundation.
[1] W. Russel, D. Saville, and W. Schowalter, Colloidal dispersions (Cambridge University Press, Cambridge, 1989).

[2] T. C. Lubensky, D. Pettey, N. Currier, and H. Stark, Phys. Rev. E 57, 610 (1998).

[3] P. Poulin, H. Stark, T. C. Lubensky, and D. A. Weitz, Science 275, 1770 (1997).

[4] S. Ramaswamy, R. Nityananda, V. Raghunathan, and J. Prost, Mol. Cryst. Liq. Cryst 288, 175 (1996).

[5] B. I. Lev and P. M. Tomchuk, Phys. Rev. E 59, 591
(1999).

[6] J. C. Loudet and P. Poulin, Phys. Rev. Lett. 87, 165503 (2001).

[7] M. Tasinkevych, N. M. Silvestre, P. Patrício, and M. M. Telo da Gama, Eur. Phys. J. E 9, 341 (2002).

[8] V. J. Anderson, E. M. Terentjev, S. P. Meeker, J. Crain, and W. C. K. Poon, Eur. Phys. J. 4, 11 (2001).

[9] V. J. Anderson and E. M. Terentjev, Eur. Phys. J. 4, 21 (2001).

[10] J. L. West, A. Glushchenko, G. Liao, Y. Reznikov, D. An- 
drienko, and M. P. Allen, Phys. Rev. E 66, 012702 (2002).

[11] S. P. Meeker, W. C. K. Poon, J. Crain, and E. M. Terentjev, Phys. Rev. E 61, R6083 (2000).

[12] V. Popa-Nita, T. J. Sluckin, and A. A. Wheeler, J. Phys. II 7, 1225 (1997).

[13] N. Schopohl and T. J. Sluckin, Phys. Rev. Lett. 59, 2582 (1987).

[14] N. Schopohl, Phys. Rev. Lett. 60, 755 (1988).

[15] A. K. Sen and D. Sullivan, Phys. Rev. A 35, 1391 (1987).

[16] P. G. de Gennes and J. Prost, The Physics of Liquid Crystals (Clarendon Press, Oxford, 1995), second, paperback ed.

[17] M. Stephen and J. Straley, Rev. Mod. Phys. 46, 617 (1974).

[18] P. G. Degennes, Mol Cryst Liquid Cryst 12, 193 (1971).

[19] P. L. George and H. Borouchaki, Delaunay triangulation and meshing : application to finite elements (Hermes,
Paris, 1998).

[20] W. H. Press, B. P. Flannery, S. A. Teukolsky, and W. T. Vetterling, Numerical Recipes in Fortran (Cambridge University Press, Cambridge, 1992), 2nd ed.

[21] D. Andrienko, M. P. Allen, G. Skacej, and S. Zumer, Phys. Rev. E 65, 041702 (2002).

[22] D. Andrienko, M. Tasinkevych, P. Patricio, M. P. Allen, and M. M. Telo da Gama (2003), cond-mat/0307261.

[23] E. Martin del Rio, M. M. Telo da Gama, E. de Miguel, and L. F. Rull, Physical Review E 52, 5028 (1995).

[24] P. J. Bos and K. R. Beran, Molecular Crystals And Liquid Crystals 113, 329 (1984).

[25] A. Sparavigna, O. D. Lavrentovich, and A. Strigazzi, Phys. Rev. E 49, 1344 (1994).

[26] A. Boulbitch, Europhysics Letters 59, 910 (2002).

[27] M. Deserno and T. Bickel, Europhysics Letters 62, 767 (2003). 